\title{
Study of Rayleigh-Bénard Magneto Convection in a Micropolar Fluid with Maxwell-Cattaneo Law
}

\author{
Subbarama Pranesh ${ }^{1}$, Rojipura V. Kiran ${ }^{2}$ \\ ${ }^{1}$ Department of Mathematics, Christ University, Bangalore, India \\ ${ }^{2}$ Department of Mathematics, Christ Junior College, Bangalore, India \\ E-mail: pranesh.s@christuniversity.in, kiran.rv@cjc.christcollege.edu \\ Received August 17, 2010; revised October 8, 2010; accepted October 12, 2010
}

\begin{abstract}
The effects of result from the substitution of the classical Fourier law by the non-classical Maxwell-Cattaneo law on the Rayleigh-Bénard Magneto-convection in an electrically conducting micropolar fluid is studied using the Galerkin technique. The eigenvalue is obtained for free-free, rigid-free and rigid-rigid velocity boundary combinations with isothermal or adiabatic temperature on the spin-vanishing boundaries. The influences of various micropolar fluid parameters are analyzed on the onset of convection. The classical approach predicts an infinite speed for the propagation of heat. The present non-classical theory involves a wave type heat transport (SECOND SOUND) and does not suffer from the physically unacceptable drawback of infinite heat propagation speed. It is found that the results are noteworthy at short times and the critical eigenvalues are less than the classical ones.
\end{abstract}

Keywords: Rayleigh-Bénard Magneto-Convection, Magnetic Field, Micropolar Fluid, Maxwell-Cattaneo Law

\section{Introduction}

The Classical Fourier law of heat conduction expresses that the heat flux within a medium is proportional to the local temperature gradient in the system. A well known consequence of this law is that heat perturbations propagates with an infinite velocity. This drawback of the classical law motivated Maxwell [1], Cattaneo [2], Lindsay and Straughan [3], Straughan and Franchi [4], Lebon and Cloot [5], Siddheshwar [6] and Pranesh [7], Dauby et al. [8] and Straughan [9] to adopt a non-classical heat flux Maxwell-Cattaneo law in studying Rayleigh-Bénard/Marangoni convection to get rid of this unphysical results. This Maxwell-Cattaneo equation contains an extra inertial term with respect to the Fourier law.

$$
\tau \frac{d \vec{Q}}{d t}+\vec{Q}=-\chi \nabla T
$$

where $\vec{Q}$ is the heat flux, $\tau$ is a relaxation time and $\chi$ is the heat conductivity. This heat conductivity equation and the conservation of energy equation introduce the hyperbolic equation, which describes heat propagation with finite speed. Puri and Jordan [10,11] and Puri and Kythe $[12,13]$ have studied other fluid mechanics problems by employing the Maxwell-Cattaneo heat flux law.
The theory of micropolar fluid is due to Eringen (see [14-16]), whose theory allows for the presence of particles in the fluid by additionally accounting for particle motion. The motivation for the study comes from many applications involving unclean fluids wherein the clean fluid is evenly interspersed with particles, which may be dust, dirt, ice or raindrops, or other additives(see [17, $18])$. This suggests geophysical or industrial convection contexts for the application of micropolar fluids. Many authors (see [19-28]) have investigated the problem of Rayleigh-Bénard convection in Eringen's micropolar fluid and concluded that the stationary convection is the preferred mode. The reported works on convection in micropolar fluid concern with classical Fourier heat flux law.

The objective of this paper is to study the RayleighBénard magneto-convection in micropolar fluid by replacing the classical Fourier law by non-classical Maxwell-Cattaneo law using Galerkin technique.

\section{Mathematical Formulation}

Consider an infinite horizontal layer of a Boussinesquian, electrically conducting fluid, with non-magnetic suspended particle, of depth'd'. Cartesian co-ordinate system is taken with origin in the lower boundary and z-axis ver- 
tically upwards. Let $\Delta \mathrm{T}$ be the temperature difference between the upper and lower boundaries. (See Figure 1)

The governing equations for the Rayleigh-Bénard situation in a Boussinesquian fluid with suspended particles are

Continuity equation:

$$
\nabla \cdot \vec{q}=0,
$$

\section{Conservation of linear momentum:}

$$
\left.\begin{array}{c}
\rho_{o}\left[\frac{\partial \vec{q}}{\partial t}+(\vec{q} . \nabla) \vec{q}\right]=-\nabla P-\rho g \hat{k}+ \\
(2 \zeta+\eta) \nabla^{2} \vec{q}+\zeta \nabla \times \vec{\omega}+\mu_{m}(\vec{H} . \nabla) \vec{H},
\end{array}\right\}
$$

\section{Conservation of angular momentum:}

$$
\left.\begin{array}{l}
\rho_{o} I\left[\frac{\partial \vec{\omega}}{\partial t}+(\vec{q} \cdot \nabla) \vec{\omega}\right]=\left(\lambda^{\prime}+\eta^{\prime}\right) \nabla(\nabla \cdot \vec{\omega})+ \\
\left(\eta^{\prime} \nabla^{2} \vec{\omega}\right)+\zeta(\nabla \times \vec{q}-2 \vec{\omega}),
\end{array}\right\}
$$

\section{Conservation of energy:}

$$
\frac{\partial T}{\partial t}+\left(\vec{q}-\frac{\beta}{\rho_{o} C_{v}} \nabla \times \vec{\omega}\right) \cdot \nabla T=-\nabla \cdot \vec{Q},
$$

Maxwell-Cattaneo heat flux law:

$$
\tau\left[\dot{\vec{Q}}+\vec{\omega}_{1} \times \vec{Q}\right]=-\vec{Q}-\kappa \nabla T,
$$

\section{Magnetic induction equation:}

$$
\frac{\partial \vec{H}}{\partial t}+(\vec{q} . \nabla) \vec{H}=(\vec{H} . \nabla) \vec{q}+\gamma_{m} \nabla^{2} \vec{H},
$$

\section{Equation of state:}

$$
\rho=\rho_{o}\left[1-\alpha\left(T-T_{o}\right)\right] .
$$

where $\vec{q}$ is the velocity, $\vec{\omega}$ is the spin, $T$ is the temperature, $P$ is the hydromagnetic pressure, $\vec{H}$ is the magnetic field, $\rho$ is the density, $\rho_{o}$ is the density of the fluid at reference temperature $T=T_{o}, g$ is the acceleration due to gravity, $\zeta$ is the coupling viscosity coefficient or vortex viscosity, $\eta$ is the shear kinematic viscosity coefficient, $I$ is the moment of inertia, $\lambda^{\prime}$ and $\eta^{\prime}$ are the bulk and shear spin viscosity coefficient, $\beta$

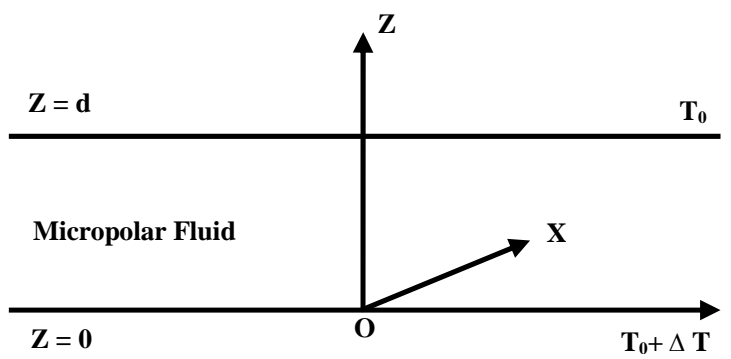

Figure 1. Schematic diagram of the Rayleigh-Bénard situation for micropolar fluid. is the micropolar heat conduction coefficient, $C_{v}$ is the specific heat, $\kappa$ is the thermal conductivity, $\alpha$ is the coefficient of thermal expansion, $\gamma_{m}=\frac{1}{\mu_{m} \sigma_{m}}$ is the magnetic viscosity $\left(\sigma_{m}\right.$ :electrical conductivity and $\mu_{m}$ : magnetic permeability), $\vec{\omega}_{1}=\frac{1}{2} \nabla \times \vec{q}, \quad \vec{Q}$ is the heat flux vector and $\tau$ is the constant relation time.

The basic state of the fluid being quiescent is described by

$$
\left.\begin{array}{l}
\vec{q}_{b}=0, \vec{\omega}_{b}=0, \vec{H}_{b}=H_{o} \hat{k}, \\
P=P_{b}(z), \rho=\rho_{b}(z), \\
\vec{Q}=\left(0,0, Q_{b}(z)\right), T=T_{b}(z)
\end{array}\right\}
$$

Equations (2), (4), (5) and (7) in the basic state specified by "(8)" respectively become

$$
\begin{aligned}
& \frac{d P_{b}}{d z}=-\rho_{o} g \hat{k}, \quad \frac{d \vec{Q}_{b}}{d z}=0, \\
& \vec{Q}_{b}=-\kappa \frac{d T_{b}}{d z}, \\
& \rho_{b}=\rho_{o}\left[1-\alpha\left(T_{b}-T_{o}\right)\right], \\
& \frac{d^{2} T_{b}}{d z^{2}}=0 .
\end{aligned}
$$

Equations (1), (3) and (6) are identically satisfied by the concerned basic state variables. We now superpose infinitesimal perturbations on the quiescent basic state and study the instability.

\section{Linear Stability Analysis}

Let the basic state be disturbed by an infinitesimal thermal perturbation. We now have

$$
\left.\begin{array}{l}
\vec{q}=\vec{q}_{b}+\vec{q}^{\prime}, \vec{\omega}=\vec{\omega}_{b}+\vec{\omega}^{\prime}, \\
P=P_{b}+P^{\prime}, \vec{Q}=\vec{Q}_{b}+\vec{Q}^{\prime}, \\
\rho=\rho_{b}+\rho^{\prime}, T=T_{b}+T^{\prime}, \\
\vec{H}=\vec{H}_{b}+\vec{H}^{\prime}
\end{array}\right\}
$$

The primes indicate that the quantities are infinitesimal perturbations and subscript ' $b$ ' indicates basic state value.

Substituting "(10)" into "(1)-(7)" and using the basic state (9), we get linearised equation governing the infinitesimal perturbations in the form

$$
\left.\begin{array}{c}
\nabla \cdot \vec{q}^{\prime}=0, \\
\rho_{o}\left[\frac{\partial \vec{q}^{\prime}}{\partial t}\right]=-\nabla P-\rho^{\prime} g \hat{k}+(2 \zeta+\eta) \nabla^{2} \vec{q}^{\prime}+ \\
\left(\zeta \nabla \times \vec{\omega}^{\prime}\right)+\mu_{m}\left(\vec{H}^{\prime} . \nabla\right) \vec{H}^{\prime},
\end{array}\right\}
$$




$$
\begin{aligned}
& \left.\begin{array}{l}
\rho_{o} I\left[\frac{\partial \vec{\omega}^{\prime}}{\partial t}\right]=\left(\lambda^{\prime}+\eta^{\prime}\right) \nabla\left(\nabla \vec{\omega}^{\prime}\right)+ \\
\left(\eta^{\prime} \nabla^{2} \vec{\omega}^{\prime}\right)+\zeta\left(\nabla \times \vec{q}^{\prime}-2 \vec{\omega}^{\prime}\right),
\end{array}\right\} \\
& \frac{\partial T^{\prime}}{\partial t}=\frac{\Delta T}{d}\left[\vec{q}^{\prime}-\frac{\beta}{\rho_{o} C_{v}} \nabla \times \vec{\omega}^{\prime}\right]-\nabla \cdot \vec{Q}^{\prime}, \\
& {\left[1+\tau \frac{\partial}{\partial t}\right] \vec{Q}^{\prime}=-\frac{1}{2} \chi_{1} \frac{\Delta T}{d}\left(\frac{\partial \vec{q}^{\prime}}{\partial z}-\nabla W^{\prime}\right)-\kappa \nabla T^{\prime},} \\
& \frac{\partial \vec{H}^{\prime}}{\partial t}=\left(H_{o} \frac{\partial W}{\partial z} \hat{k}\right)+\gamma_{m} \nabla^{2} \vec{H}^{\prime}, \\
& \rho^{\prime}=-\alpha \rho_{0} T^{\prime} .
\end{aligned}
$$

Operating divergence on the "(15)" and substituting in “(14)”, on using “(11)”, we get

$$
\left.\begin{array}{c}
\left(1+\tau \frac{\partial}{\partial t}\right) \frac{\partial T^{\prime}}{\partial t}=\left(1+\tau \frac{\partial}{\partial t}\right) \frac{\Delta T}{d} \\
{\left[W^{\prime}-\frac{\beta}{\rho_{o} C_{v}} \Omega_{z}\right]+\kappa \nabla^{2} T^{\prime}-} \\
\frac{1}{2} \chi_{1} \frac{\Delta T}{d}\left(\nabla^{2} W^{\prime}\right),
\end{array}\right\}
$$

where $\Omega=\nabla \times \vec{\omega}^{\prime}$. The perturbation "(12), (13), (16) and (18)" are non-dimensionalised using the following definition:

$$
\left.\begin{array}{l}
\left(x^{*}, y^{*}, z^{*}\right)=\frac{(x, y, z)}{d}, W^{*}=\frac{W^{\prime}}{(\kappa / d)}, \\
\vec{\omega}^{*}=\frac{\omega^{\prime}}{\left(\kappa / d^{2}\right)}, t^{*}=\frac{t}{\left(d^{2} / \kappa\right)}, \\
T^{*}=\frac{T^{\prime}}{\Delta T}, H^{*}=\frac{\vec{H}^{\prime}}{H_{o}}, \Omega^{*}=\frac{\Omega_{z}}{\left(\kappa / d^{3}\right)}
\end{array}\right\}
$$

Using "(17)" in "(12)", Operating curl twice on the resulting equation, operating curl once on "(13)" and non-dimensionalising the two resulting equations and also "(16)-(18)".

$$
\begin{gathered}
\frac{1}{\operatorname{Pr}} \frac{\partial}{\partial t}\left(\nabla^{2} W\right)=R \nabla_{1}^{2} T+ \\
\left(1+N_{1}\right) \nabla^{4} W+N_{1} \nabla^{2} \Omega_{z}+ \\
Q \frac{\operatorname{Pr}}{\operatorname{Pm}} \nabla^{2}\left(\frac{\partial H_{z}}{\partial z}\right) \\
\frac{N_{2}}{\operatorname{Pr}} \frac{\partial}{\partial t}\left(\Omega_{z}\right)=N_{3} \nabla^{2} \Omega_{z}-N_{1} \nabla^{2} W-2 N_{1} \Omega_{z}
\end{gathered}
$$

$$
\left.\begin{array}{c}
\frac{\partial H_{z}}{\partial z}=\frac{\partial W}{\partial z}+\frac{\operatorname{Pr}}{P m} \nabla^{2} H_{z} \\
\left(1+2 C \frac{\partial}{\partial t}\right) \frac{\partial T}{\partial t}=\left(1+2 C \frac{\partial}{\partial t}\right) W- \\
\left(1+2 C \frac{\partial}{\partial t}\right) N_{5} \Omega_{z}+\nabla^{2} T-C \nabla^{2} W
\end{array}\right\}
$$

where the asterisks have been dropped for simplicity and the non-dimensional parameters $N_{1}, N_{3}, N_{5}, R, Q, \operatorname{Pr}$, $P m$ and $C$ are as defined as

$$
\begin{array}{lr}
N_{1}=\frac{\zeta}{\zeta+\eta} & \text { (Coupling Parameter) } \\
N_{3}=\frac{\eta^{\prime}}{(\zeta+\eta) d^{2}} & \text { (Couple Stress Parameter) }
\end{array}
$$
$N_{5}=\frac{\beta}{\rho_{o} C_{v} d^{2}}$ (Micropolar Heat Conduction Parameter)

$$
\begin{array}{lr}
\operatorname{Pr}=\frac{\zeta+\eta}{\rho_{o} \kappa} & \text { (Prandtl Number) } \\
\operatorname{Pm}=\frac{\zeta+\eta}{\gamma_{m}} & \text { (Magnetic Prandtl Number) } \\
R=\frac{\rho_{o} \alpha g \Delta T d^{3}}{\kappa(\zeta+\eta)} & \text { (Rayleigh Number) } \\
Q=\frac{\mu_{m} \vec{H}_{o} d^{2}}{\gamma_{m}(\zeta+\eta)} & \text { (Chandrasekhar Number) } \\
C=\frac{\tau \kappa}{2 d^{2}} & \text { (Cattaneo Number) }
\end{array}
$$

The infinitesimal perturbation $W, \Omega_{z}$ and $T$ are assumed to be periodic waves (see Chandrasekhar 1961) and hence these permit a normal mode solution in the form

$$
\left[\begin{array}{l}
W \\
\Omega_{z} \\
H_{z} \\
T
\end{array}\right]=\left[\begin{array}{l}
W(z) \cdot e^{i((x+m y)} \\
G(z) \cdot e^{i(x+m y)} \\
H_{z}(z) \cdot e^{i(l x+m y)} \\
T(z) \cdot e^{i(l x+m y)}
\end{array}\right]
$$

where $l$ and $m$ are horizontal components of the wave number $\vec{a}$, Substituting "(24)" into "(20)-(23)", we get

$$
\left.\begin{array}{l}
\left(1+N_{1}\right)\left(D^{2}-a^{2}\right)^{2} W- \\
R a^{2} T+N_{1}\left(D^{2}-a^{2}\right) G+ \\
Q \frac{\operatorname{Pr}}{P m}\left(D^{2}-a^{2}\right) H_{z}
\end{array}\right\}=0
$$




$$
\begin{aligned}
& \left.\begin{array}{r}
N_{3}\left(D^{2}-a^{2}\right) G- \\
N_{1}\left(D^{2}-a^{2}\right) W-2 N_{1} G
\end{array}\right\}=0 \\
& \left.D W+\frac{P r}{P m}\left(D^{2}-a^{2}\right) H_{z}\right\}=0 \\
& \left.\begin{array}{c}
W-N_{5} G+\left(D^{2}-a^{2}\right) T- \\
C\left(D^{2}-a^{2}\right) W
\end{array}\right\}=0
\end{aligned}
$$

where $D=\frac{d}{d z}$.

The set of ordinary differential "(25)-(28)" are approximations based on physical considerations to the system of partial differential "(20)-(23)". Although the relationship between the solutions of the governing partial differential equations and the corresponding ordinary differential equations has not been established, these linear models reproduce qualitatively the convective phenomena observable through the full system.

Eliminating $H_{z}$ between "(25) and (27)", we get

$$
\left.\begin{array}{c}
\left(1+N_{1}\right)\left(D^{2}-a^{2}\right)^{2} W-R a^{2} T+ \\
N_{1}\left(D^{2}-a^{2}\right) G-Q D^{2} W
\end{array}\right\}=0
$$

We now apply the Galerkin method to "(26), (28) and (29)" that gives general results on the eigen value of the problem using simple, polynomial, trial functions for the lowest eigen value. We obtain an approximate solution of the differential equations with the given boundary conditions by choosing trial functions for velocity, microrotation and temperature perturbations that satisfy the boundary conditions but may not exactly satisfy the differential equations. This leads to residuals when the trial functions are substituted into the differential equations. The Galerkin method requires the residual to be orthogonal to each individual trial function.

In the Galerkin procedure, we expand the velocity, microrotation and temperature by,

$$
\begin{aligned}
& W(z, t)=\sum A_{i}(t) W_{i}(z), \\
& G(z, t)=\sum B_{i}(t) G_{i}(z), \\
& T(z, t)=\sum E_{i}(t) T_{i}(z)
\end{aligned}
$$

where $W_{i}(z), G_{i}(z)$ and $T_{i}(z)$ are polynomials in $z$ that generally have to satisfy the given boundary conditions.

For the single term Galerkin expansion technique we take $i=j=1$. Multiplying "(29)" by $W$, "(26)" by $G$ and "(28)" by $T$, integrating the resulting equation with respect to $\mathrm{z}$ from 0 to 1 and taking $W=A W_{1}, G=B G_{1}$ and $T=E T_{1}$ in which $A, B$ and $E$ are constants with $W_{1}, G_{1}$ and $T_{1}$ are trial functions. This procedure yields the following equation for the Rayleigh number $R$ :

$$
R=\frac{\left\langle T_{1}\left(D^{2}-a^{2}\right) T_{1}\right\rangle\left[Y_{1} \cdot Y_{2}+N_{1}^{2} \cdot Y_{3}\right]}{a^{2}\left\langle W_{1} T_{1}\right\rangle Y_{4}}
$$

where

$$
\begin{aligned}
Y_{1}= & \left(1+N_{1}\right)\left\langle W_{1}\left(D^{2}-a^{2}\right)^{2} W_{1}\right\rangle- \\
& Q\left\langle W_{1} D^{2} W_{1}\right\rangle, \\
Y_{2}= & N_{3}\left\langle G_{1}\left(D^{2}-a^{2}\right) G_{1}\right\rangle-2 N_{1}\left\langle G_{1}^{2}\right\rangle, \\
Y_{3}= & \left\langle G_{1}\left(D^{2}-a^{2}\right) W_{1}\right\rangle\left\langle W_{1}\left(D^{2}-a^{2}\right) G_{1}\right\rangle, \\
Y_{4}= & N_{1} N_{5}\left\langle G_{1}\left(D^{2}-a^{2}\right) W_{1}\right\rangle\left\langle T_{1} G_{1}\right\rangle+ \\
& Y_{2}\left[C .\left\langle T_{1}\left(D^{2}-a^{2}\right) W_{1}\right\rangle-\left\langle T_{1} W_{1}\right\rangle\right]
\end{aligned}
$$

In the "(30)", $\langle---\rangle$ denotes integration with respect to $z$ between $z=0$ and $z=1$. We note here that $R$ in equation (30) is a functional and the Euler-Lagrange equations for the extremisation of R are "(26), (28) and (29)".

The value of critical Rayleigh number depends on the boundaries. In this paper we consider various boundary combinations and these are discussed below.

1) Free - Free isothermal/adiabatic, no spin.

2) Rigid - Rigid isothermal/adiabatic, no spin.

3) Rigid - Free isothermal/adiabatic, no spin.

Critical Rayleigh number for free-free isothermal boundaries, No spin:

The boundary conditions are

$$
W=D^{2} W=T=G=0, \quad \text { at } \quad z=0,1 .
$$

The trial functions satisfying "(30)" are

$$
\left.\begin{array}{l}
W_{1}=z^{4}-2 z^{3}+z, \\
T_{1}=z(1-z), \\
G_{1}=z(1-z)
\end{array}\right\}
$$

Substituting "(32)" in "(30)" and performing the integration, we get

$$
R=\frac{\left(10+a^{2}\right) M_{1}}{51 a^{2}}
$$

where

$$
\begin{aligned}
& M_{1}=\frac{28 \cdot K_{2}\left[\left(1+N_{1}\right) K_{1}+306 Q\right]-3 N_{1}^{2} \cdot K_{3}^{2}}{K_{2}\left[C \cdot K_{3}+17\right]-N_{1} \cdot N_{5} \cdot K_{3}}, \\
& K_{1}=3024+612 a^{2}+31 a^{4}, \\
& K_{2}=N_{3}\left(10+a^{2}\right)+2 N_{1}, \\
& K_{3}=168+17 a^{2} .
\end{aligned}
$$

$R$ attains its minimum value $R_{c}$ at $a=a_{c}$.

Critical Rayleigh number for rigid-rigid isothermal boundaries, No spin: 
The boundary conditions are

$$
W=D W=T=G=0, \text { at } z=0,1 .
$$

The trial functions satisfying “(34)” are

$$
\begin{aligned}
& W_{1}=z^{4}-2 z^{3}+z^{2}, \\
& T_{1}=z(1-z), \\
& G_{1}=z(1-z)
\end{aligned}
$$

Substituting "(35)" in "(30)" and performing the integration, we get

$$
R=\frac{\left(10+a^{2}\right) M_{1}}{9 a^{2}}
$$

where

$$
\begin{aligned}
& M_{1}=\frac{28 \cdot K_{2}\left[\left(1+N_{1}\right) K_{1}+12 Q\right]-3 N_{1}^{2} \cdot K_{3}^{2}}{K_{2}\left[C \cdot K_{3}+3\right]-N_{1} \cdot N_{5} \cdot K_{3}}, \\
& K_{1}=504+24 a^{2}+a^{4}, \\
& K_{2}=N_{3}\left(10+a^{2}\right)+2 N_{1}, \\
& K_{3}=28+3 a^{2} .
\end{aligned}
$$

$R$ attains its minimum value $R_{c}$ at $a=a_{c}$.

\section{Critical Rayleigh number for rigid-free isothermal} boundaries, No spin:

The boundary conditions are

$$
\left.\begin{array}{lll}
W=D W=T=G=0, & \text { at } & z=0 \\
W=D^{2} W=T=G=0, & \text { at } & z=1
\end{array}\right\}
$$

The trial functions satisfying “(37)” are

$$
\left.\begin{array}{l}
W_{1}=2 z^{4}-5 z^{3}+3 z^{2}, \\
T_{1}=z(1-z), \\
G_{1}=z(1-z)
\end{array}\right\}
$$

Substituting "(38)" in "(30)" and performing the integration, we get

$$
R=\frac{\left(10+a^{2}\right) M_{1}}{39 a^{2}}
$$

where

$$
\begin{aligned}
& M_{1}=\frac{28 \cdot K_{2}\left[\left(1+N_{1}\right) K_{1}+216 Q\right]-3 N_{1}^{2} \cdot K_{3}^{2}}{K_{2}\left[C \cdot K_{3}+13\right]-N_{1} \cdot N_{5} \cdot K_{3}}, \\
& K_{1}=4536+432 a^{2}+19 a^{4}, \\
& K_{2}=N_{3}\left(10+a^{2}\right)+2 N_{1}, \\
& K_{3}=126+13 a^{2} .
\end{aligned}
$$

$R$ attains its minimum value $R_{c}$ at $a=a_{c}$.

Critical Rayleigh number for free-free adiabatic boundaries, No spin:
The boundary conditions are

$$
W=D^{2} W=D T=G=0, \text { at } z=0,1 .
$$

The trial functions satisfying "(40)" are

$$
\left.\begin{array}{l}
W_{1}=z^{4}-2 z^{3}+z, \\
T_{1}=1, \\
G_{1}=z(1-z)
\end{array}\right\}
$$

Substituting "(41)" in "(30)" and performing the integration, we get

$$
R=\left(\frac{5 M_{1}}{42}\right)
$$

where

$$
\begin{aligned}
& M_{1}=\frac{28 \cdot K_{2}\left[\left(1+N_{1}\right) K_{1}+306 Q\right]-3 N_{1}^{2} \cdot K_{3}^{2}}{84 K_{2}\left[C \cdot\left(10+a^{2}\right)+1\right]-5 N_{1} \cdot N_{5} \cdot K_{3}}, \\
& K_{1}=3024+612 a^{2}+31 a^{4}, \\
& K_{2}=N_{3}\left(10+a^{2}\right)+2 N_{1}, \\
& K_{3}=168+17 a^{2} .
\end{aligned}
$$

$R$ attains its minimum value $R_{c}$ at $a=a_{c}$.

\section{Critical Rayleigh number for rigid-rigid adiabatic} boundaries, No spin:

The boundary conditions are

$$
W=D W=D T=G=0, \text { at } z=0,1 .
$$

The trial functions satisfying "(43)" are

$$
\left.\begin{array}{l}
W_{1}=z^{4}-2 z^{3}+z^{2}, \\
T_{1}=1, \\
G_{1}=z(1-z)
\end{array}\right\}
$$

Substituting "(44)" in "(30)" and performing the integration, we get

$$
R=\left(\frac{30 M_{1}}{42}\right)
$$

where

$$
\begin{aligned}
& M_{1}=\frac{28 \cdot K_{2}\left[\left(1+N_{1}\right) K_{1}+12 Q\right]-3 N_{1}^{2} \cdot K_{3}^{2}}{14 K_{2}\left[C \cdot a^{2}+1\right]-5 N_{1} \cdot N_{5} \cdot K_{3}}, \\
& K_{1}=504+24 a^{2}+a^{4} \\
& K_{2}=N_{3}\left(10+a^{2}\right)+2 N_{1}, \\
& K_{3}=28+3 a^{2} .
\end{aligned}
$$

$R$ attains its minimum value $R_{c}$ at $a=a_{c}$.

Critical Rayleigh number for rigid-free adiabatic boundaries, No spin:

The boundary conditions are 


$$
\left.\begin{array}{lll}
W=D W=D T=G=0, & \text { at } & z=0 \\
W=D^{2} W=D T=G=0, & \text { at } & z=1
\end{array}\right\}
$$

The trial functions satisfying "(46)” are

$$
\left.\begin{array}{l}
W_{1}=2 z^{4}-5 z^{3}+3 z^{2}, \\
T_{1}=1, \\
G_{1}=z(1-z)
\end{array}\right\}
$$

Substituting “(47)" in "(30)" and performing the integration, we get

$$
R=\left(\frac{20 M_{1}}{126}\right)
$$

where

$$
\begin{aligned}
& M_{1}=\frac{28 \cdot K_{2}\left[\left(1+N_{1}\right) K_{1}+216 Q\right]-3 N_{1}^{2} \cdot K_{3}^{2}}{21 K_{2}\left[C \cdot\left(20+3 a^{2}\right)+1\right]-5 N_{1} \cdot N_{5} \cdot K_{3}}, \\
& K_{1}=4536+432 a^{2}+19 a^{4}, \\
& K_{2}=N_{3}\left(10+a^{2}\right)+2 N_{1}, \\
& K_{3}=126+13 a^{2} .
\end{aligned}
$$

$R$ attains its minimum value $R_{c}$ at $a=a_{c}$.

\section{Results and Discussions}

In this paper we study the onset of Rayleigh-Bénard Magneto Convection in a micropolar fluid by replacing the classical Fourier heat flux law by non-classical Maxwell-Cattaneo law.

Figures 2 (a)-(c) is the plot of critical Rayleigh number $\mathrm{R}_{\mathrm{c}}$ versus coupling parameter $N_{1}$, Couple stress parameter $N_{3}$ and micropolar heat conduction parameter $N_{5}$ respectively for different values of Cattaneo number $C$ and Chandrasekhar number $Q$ for free-free isothermal, no- spin boundary conditions. It is observed that as $N_{1}$ and $N_{5}$ increases, $R_{c}$ increases, that is an increase in $N_{1}$ and $N_{5}$ is to stabilize the system. The increase in $N_{3}$ decreases $R_{c}$, that is increase in $N_{3}$ destabilizes the system. Why and how micropolar parameters $N_{1}, N_{3}$ and $N_{5}$ stabilizes/destabilizes the system are given in the Table 1. Figures 3-4 are the plot of $R_{c}$ versus $N_{1}, N_{3}$ and $N_{5}$ for rigid-rigid and rigid-free velocity boundary combination with isothermal temperature and spin vanishing boundaries. Figures 5-7 are the plot of $R_{c}$ verses $N_{1}, N_{3}$ and $N_{5}$ for free-free, rigid-rigid and rigid-free velocity boundary combination respectively, with adiabatic temperature and spin vanishing boundaries. The results of these graphs, i.e., the effects of $N_{1}, N_{3}$ and $N_{5}$ on the onset of convection are qualitatively similar to free-free isothermal case.

It is also observed from the above figures that the increase in $Q$ increases the $R_{c}$, from this we conclude that

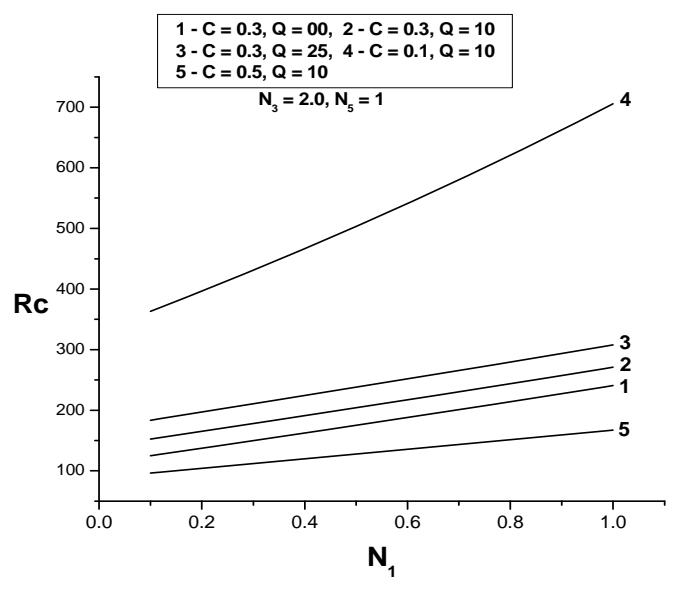

(a)

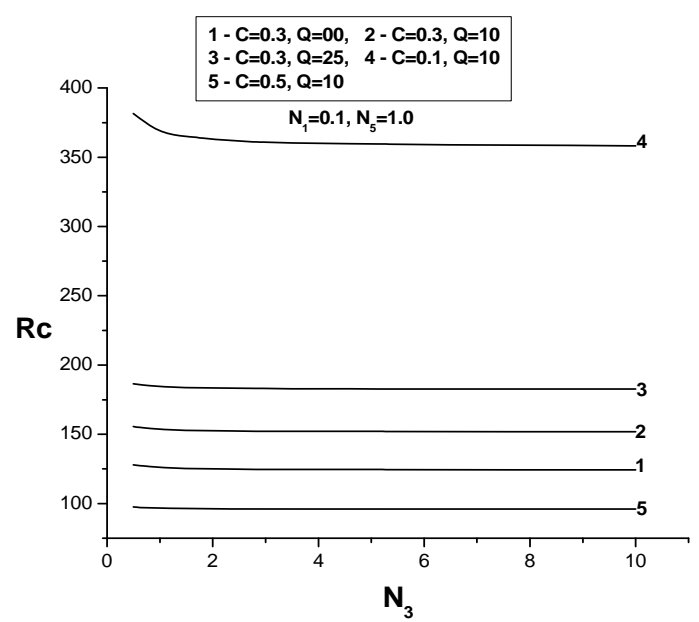

(b)

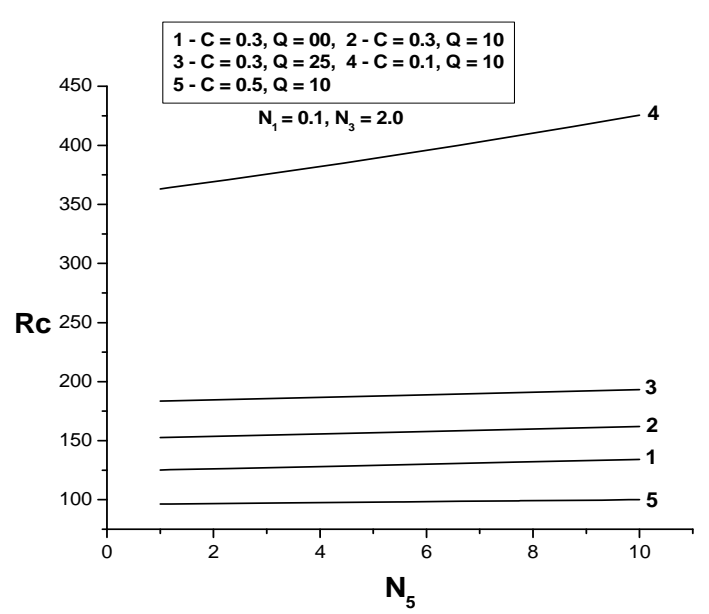

(c)

Figure 2. Plot of critical Rayleigh number $\boldsymbol{R}_{c}$ Vs. (a) coupling parameter $N_{1}$, (b) couple stress parameter $N_{3}$, (c) coupling parameter $N_{5}$ with respect to free-free isothermal no spin boundary condition for different values Chandrasekhar number $Q$ and different values of Cattaneo number C. 


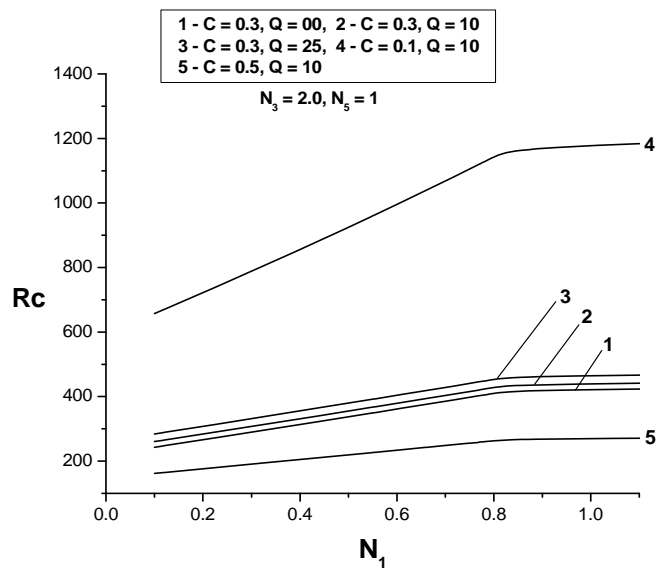

(a)

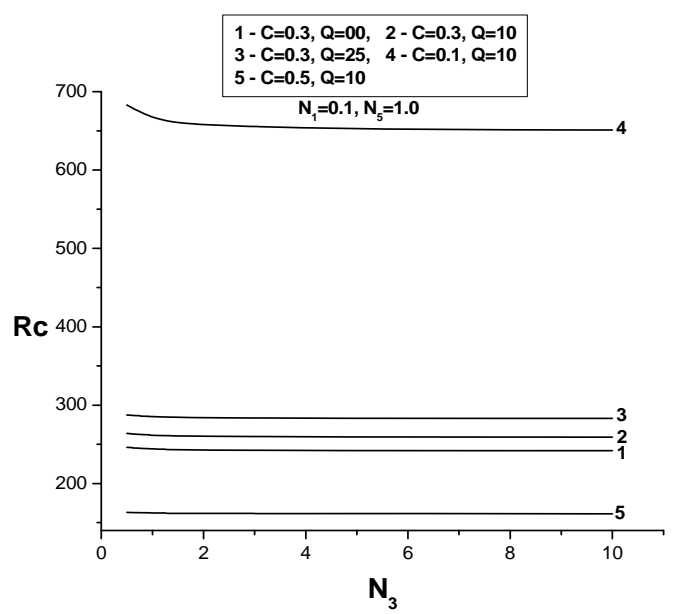

(b)

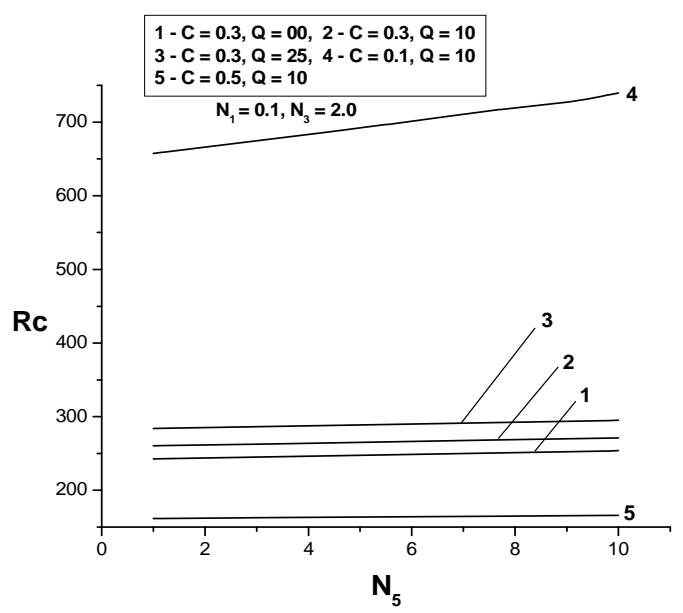

(c)

Figure 3. Plot of critical Rayleigh number $\boldsymbol{R}_{c}$ Vs. (a) coupling parameter $N_{1}$, (b) couple stress parameter $N_{3}$, (c) coupling parameter $N_{5}$ with respect to rigid-rigid isothermal no spin boundary condition for different values Chandrasekhar number $Q$ and different values of Cattaneo number $C$.

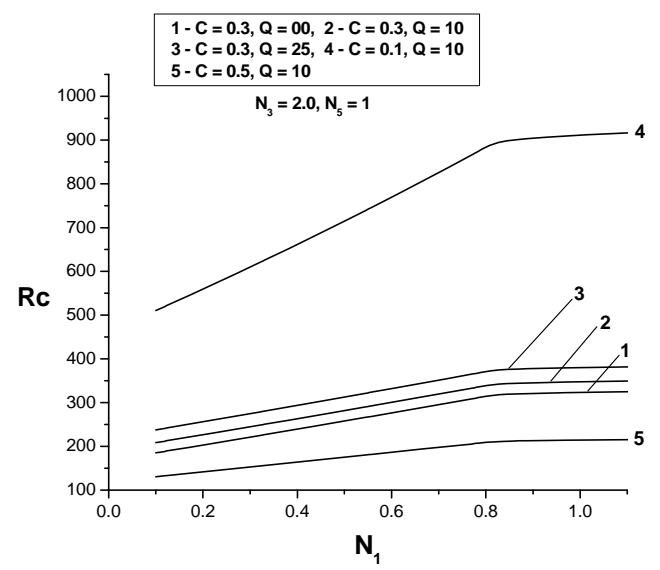

(a)

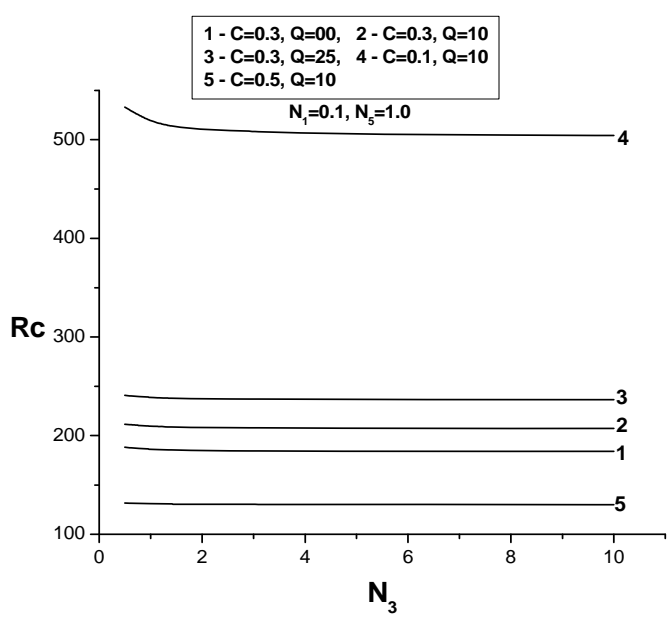

(b)

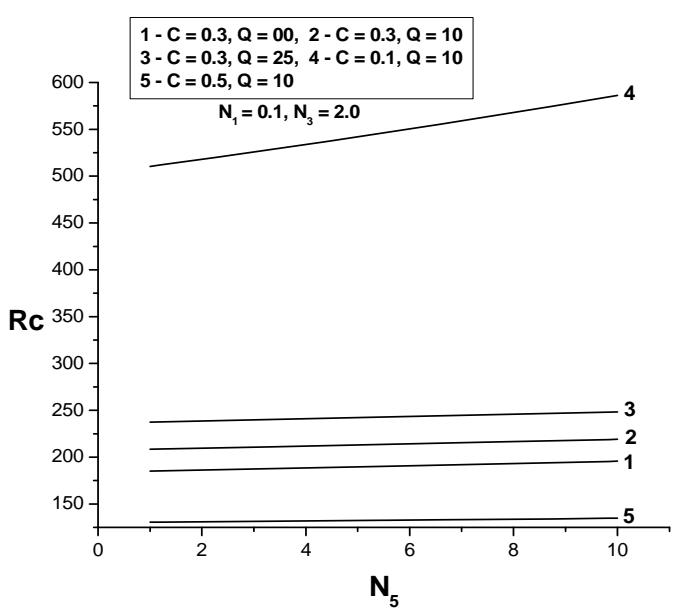

(c)

Figure 4. Plot of critical Rayleigh number $\boldsymbol{R}_{c}$ Vs. (a) coupling parameter $N_{1}$, (b) couple stress parameter $N_{3}$, (c) coupling parameter $N_{5}$ with respect to rigid-free isothermal no spin boundary condition for different values Chandrasekhar number $Q$ and different values of Cattaneo number C. 


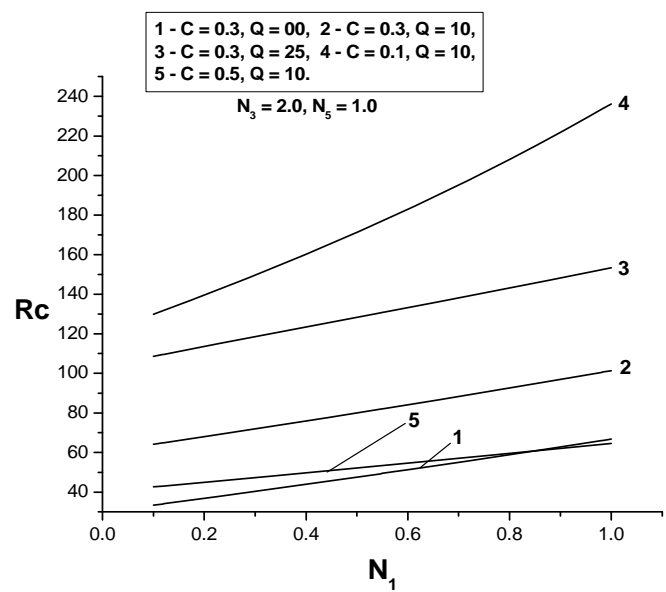

(a)

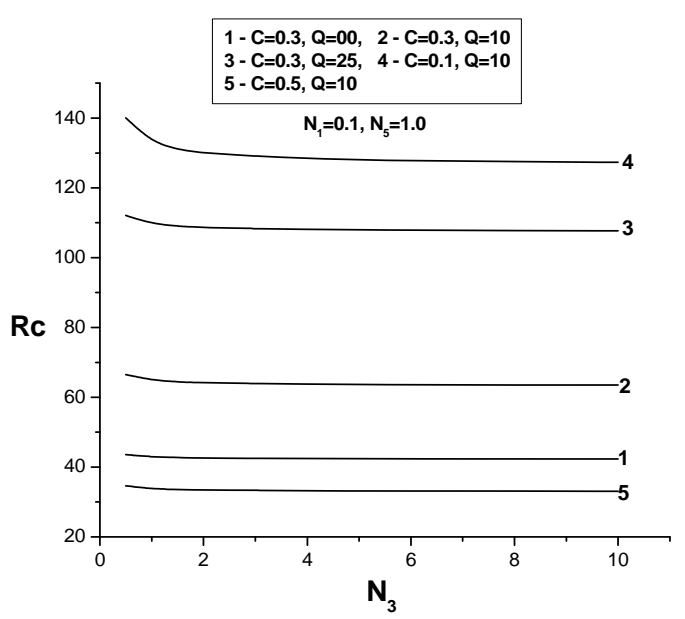

(b)

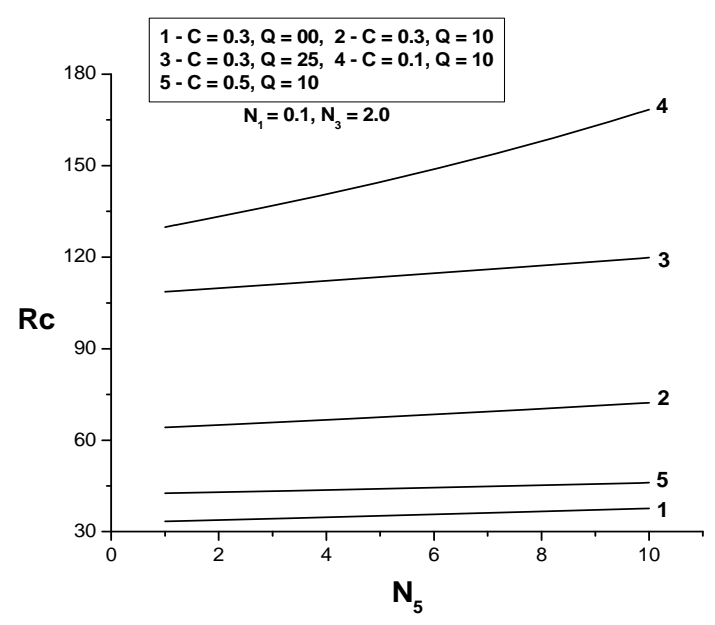

(c)

Figure 5. Plot of critical Rayleigh number $R_{c}$ Vs. (a) coupling parameter $N_{1}$, (b) couple stress parameter $N_{3}$, (c) coupling parameter $N_{5}$ with respect to free-free adiabatic no spin boundary condition for different values Chandrasekhar number $Q$ and different values of Cattaneo number C.

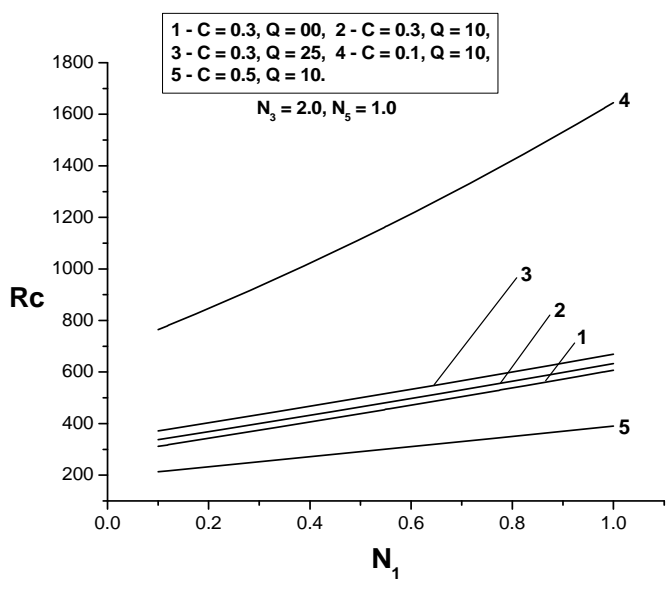

(a)

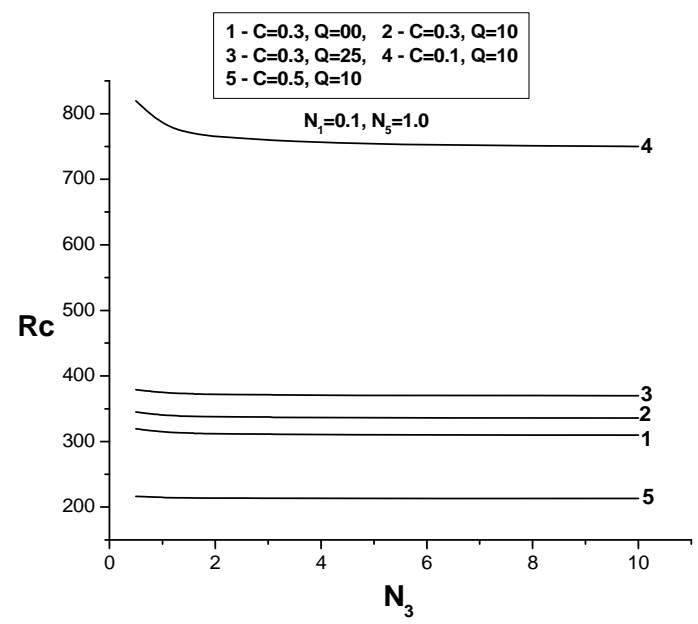

(b)

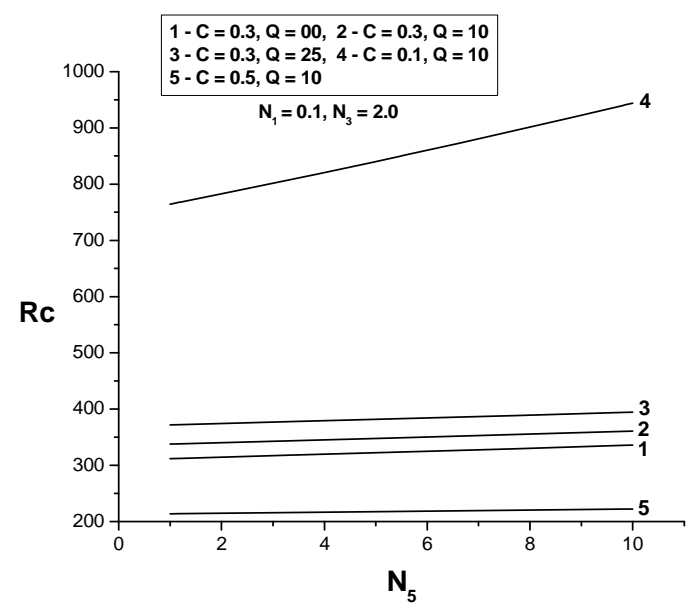

(c)

Figure 6. Plot of critical Rayleigh number $R_{c}$ Vs. (a) coupling parameter $N_{1}$, (b) couple stress parameter $N_{3}$, (c) coupling parameter $N_{5}$ with respect to rigid-rigid adiabatic no spin boundary condition for different values Chandrasekhar number $Q$ and different values of Cattaneo number C. 


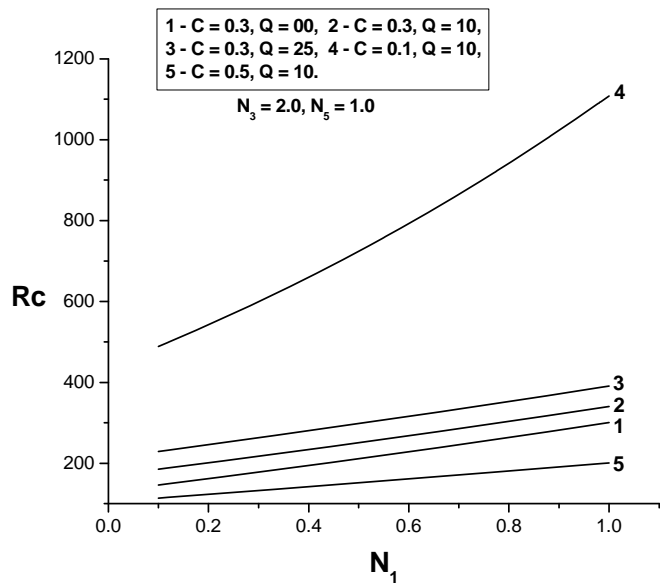

(a)

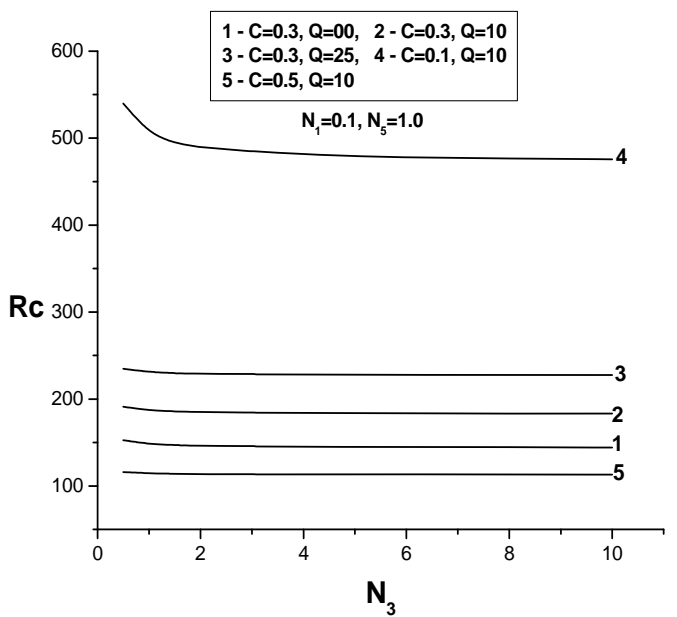

(b)

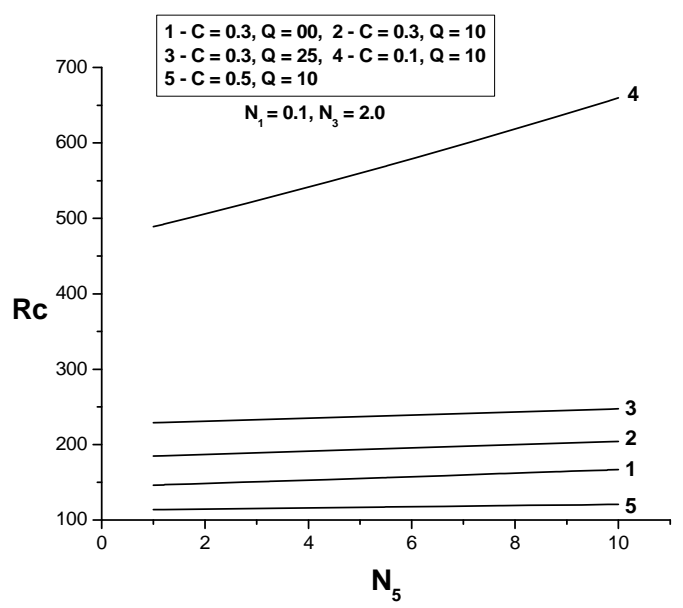

(c)

Figure 7. Plot of critical Rayleigh number $\boldsymbol{R}_{c}$ Vs. (a) coupling parameter $N_{1}$, (b) couple stress parameter $N_{3}$, (c) coupling parameter $N_{5}$ with respect to rigid-free adiabatic no spin boundary condition for different values Chandrasekhar number $Q$ and different values of Cattaneo number C.
Table 1. Why and how of the stabilizing/destabilizing effects of the micropolar fluid parameters $N_{1} N_{3} N_{5}$.

\begin{tabular}{|c|c|c|}
\hline Parameter & Nature of effect & Physical reason \\
\hline $\begin{array}{c}N_{1} \\
0 \leq N_{1} \leq 1\end{array}$ & $\begin{array}{c}\text { Stabilizing } \\
\text { (as } N_{1} \text { increases) }\end{array}$ & $\begin{array}{l}\text { Increase in } N_{1} \text { indicates the in- } \\
\text { crease in the concentration of } \\
\text { microelements. These elements } \\
\text { consume the greater part of the } \\
\text { energy of the system in develop- } \\
\text { ing the gyrational velocities of the } \\
\text { fluid and as a result the onset of } \\
\text { convection is delayed. }\end{array}$ \\
\hline $\begin{array}{c}\quad N_{3} \\
0 \leq N_{3} \leq \mathrm{m} \\
\text { (m: finite, real) }\end{array}$ & $\begin{array}{c}\text { Destabilizing } \\
\text { (as } N_{3} \text { increases) }\end{array}$ & $\begin{array}{l}\text { Increase in } N_{3} \text {, decreases the } \\
\text { couple stress of the fluid which } \\
\text { causes a decrease in microrotation } \\
\text { and hence makes the system more } \\
\text { unstable. }\end{array}$ \\
\hline $\begin{array}{c}\quad N_{5} \\
0 \leq N_{5} \leq \mathrm{n} \\
(\mathrm{n}: \text { finite, real) }\end{array}$ & $\begin{array}{c}\text { Stabilizing } \\
\text { (as } N_{5} \text { increases) }\end{array}$ & $\begin{array}{l}\text { When } N_{5} \text { increases, the heat in- } \\
\text { duced into the fluid due to these } \\
\text { microelements also increases, thus } \\
\text { reducing the heat transfer from } \\
\text { bottom to top. }\end{array}$ \\
\hline $\begin{array}{c}C \\
C \in[0,1]\end{array}$ & $\begin{array}{l}\text { Destabilizing } \\
\text { (as } C \text { increases) }\end{array}$ & $\begin{array}{l}\text { It is a scaled relaxation time and } \\
\text { hence it accelerates the onset of } \\
\text { convection. }\end{array}$ \\
\hline
\end{tabular}

the $Q$ has stabilizing effect on the system. When the magnetic field strength permeating the medium is considerably strong, it induces viscosity into the fluid, and the magnetic lines are distorted by convection. Then these magnetic lines hinder the growth of disturbances, leading to the delay in the onset of instability. However, the viscosity produced by the magnetic field lessens the rotation of the fluid particles, thus controlling the stabilizing effect of $N_{1}$.

From the figures it is observed that $C$ which represents Cattaneo number has a destabilizing influence. Increase in Cattaneo number leads to narrowing of the convection cells and thus lowering of the critical Rayleigh number. It is also observed from the figures that influence of Cattaneo number is dominant for small values because the convection cells have fixed aspect ratio.

\section{Conclusions}

Following conclusions are drawn from the problem:

1) Rayleigh-Bénard convection in Newtonian fluids may be delayed by adding micron sized suspended particles.

2) By adjusting the Chandrasekhar number $Q$ we can control the convection.

3) We can conclude the following for stationary convection in micropolar fluids

$$
R_{c}^{H H E}<R_{c}^{P H E},
$$

where HHE - Hyperbolic heat equation and PHE - Parabolic heat equation. 
4) We also find that

$$
R_{c}^{R R}>R_{c}^{R F}>R_{c}^{F F}
$$

where the superscripts correspond to the three different velocity boundary combinations. The above qualitative results are true for both isothermal and adiabatic boundaries.

5) The non-classical Maxwell-Cattaneo heat flux law involves a hyperbolic type heat transport equation that predicts finite speeds of heat wave propagation (see [29]). Hence it does not suffer from the physically unacceptable draw back of infinite heat propagation speed predicted by the parabolic heat equation. The classical Fourier flux law overpredicts the critical Rayleigh number compared to that predicted by the non-classical law.

\section{Acknowledgement}

The authors like to thank management of Christ University for their support in completing this work. Second author Kiran would like to thank Principal, Christ Junior College for deputing him for Ph.D.

\section{References}

[1] J. C. Maxwell, "On the Dynamical Theory of Gases," The Philosophical Transactions of the Royal Society, Vol. 157, 1867, pp. 49-88.

[2] C. Cattaneo, "Sulla Condizione Del Calore," Atti Del Semin. Matem. E Fis. Della Univ. Modena, Vol. 3, 1948, pp. 83-101.

[3] K. A. Lindsay and B. Straughan, "Acceleration Waves and Second Sound in a Perfect Fluid," Archive for Rational Mechanics and Analysis, Vol.68, 1978, pp 53-87.

[4] B. Straughan and F. Franchi, "Bénard Convection and the Cattaneo Law of Heat Conduction," Proceedings of the Royal Society of Edinburgh, Vol. 96A, 1984, pp. 175178.

[5] G. Lebon and A. Cloot, "A Nonlinear Stability Analysis of the Bénard-Marangoni Problem," Journal of Fluid Mechanics, Vol. 145, 1984, pp. 447-469.

[6] P. G. Siddheshwar, "Rayleigh Benard Convection in a Second Order Ferromagnetic Fluid with Second Sound," Proceedings of 8th Asian Congress of Fluid Mechanics, Shenzen, December 6-10, 1999, p. 631.

[7] S. Pranesh, "Effect of Second Sound on the Onset of Rayleigh-Bénard Convection in a Coleman-Noll Fluid," Mapana Journal of Science, Vol. 7, No. 2, 2008, pp. 1-9.

[8] P. C. Dauby, M. Nelis and G. Lebon, "Generalized Fourier Equations and Thermoconvective Instabilities," Revista Mexicana de Fisica, Vol. 48, 2001, pp. 57-62.

[9] B. Straughan, "Oscillatory Convection and the Cattaneo Law of Heat Conduction," Ricerche di Matematica, Vol. 58, No. 2, 2009, pp. 157-162.

[10] P. Puri and P. M. Jordan, “Stokes' First Problem for a
Dipolar Fluid with Nonclassical Heat Conduction," Journal of Engineering Mathematics, Vol. 36, No. 3, 1999, pp. 219-240.

[11] P. Puri and P. M. Jordan, "Wave Structure in Stokes Second Problem for Dipolar Fluid with Nonclassical Heat Conduction," Acta Mechanica, Vol. 133, No. 1-4, 1999, pp. 145-160.

[12] P. Puri and P. K. Kythe, "Nonclassical Thermal Effects in Stokes Second Problem," Acta Mechanica, Vol. 112, No. 1-4, 1995, pp. 1-9.

[13] P. Puri and P. K. Kythe, "Discontinuities in Velocity Gradients and Temperature in the Stokes First Problem with Nonclassical Heat Conduction," Quarterly of Applied Mathematics, Vol. 55, No. 1, March 1997, pp. $167-$ 176.

[14] A. C. Eringen, "Simple Microfluids," International Journal of Engineering Science, Vol. 2, No. 2, 1964, pp. 205-217.

[15] A. C. Eringen, "Theory of Thermomicrofluids," Journal of Mathematical Analysis and Applications, Vol. 38, No. 2, May 1972, pp. 480-496.

[16] A. C. Eringen, "Microcontinuum Field Theory," Springer Verlag, New York, 1999.

[17] H. Power, "Bio-Fluid Mechanics, Advances in Fluid Mechanics,” W. I. T. Press, U. K., 1995.

[18] G. Lukaszewicz, "Micropolar Fluid Theory and Applications," Birkhauser Boston, Boston, 1999.

[19] G. Ahmadi, "Stability of a Micropolar Fluid Layer Heated from Below," International Journal of Engineering Science, Vol. 14, No. 1, January 1976, pp. 81-89.

[20] Datta and V. U. K. Sastry, "Thermal Instability of a Horizontal Layer of Micropolar Fluid Heated from Below," International Journal of Engineering Science, Vol. 14, No. 7, 1976, pp. 631-637.

[21] A. Perez Garcia and J. M. Rubi, "On the Possibility of Overstable Motions of Micropolar Fluids Heated from Below," International Journal of Engineering Science, Vol. 20, No. 7, 1982, pp. 873-878.

[22] L. E. Payne and B. Straughan, "Critical Rayleigh Numbers for Oscillatory and Nonlinear Convection in an Instropic Thermomicropolar Fluid," International Journal of Engineering Science, Vol. 27, 1989, p. 827.

[23] G. Lebon and C. Perez-Garcia, "Convective Instability of a Micropolar Fluid Layer by the Method of Energy," International Journal of Engineering Science, Vol. 19, No. 10, 1981, pp. 1321-1329.

[24] S. Pranesh, "Effects of Suction-Injection-Combination (SIC) on the Onset of Rayleigh-Bénard Magnetoconvection in a Fluid with Suspended Particles," International Journal of Engineering Science, Vol. 41, No. 15, September 2003, pp. 1741-1766.

[25] P. G. Siddheshwar and S. Pranesh, "Effects of a NonUniform Basic Temperature Gradient on RayleighBénard Convection in a Micropolar Fluid," International Journal of Engineering Science, Vol. 36, No. 11, September 1998, pp. 1183- 1196.

[26] P. G. Siddheshwar and S. Pranesh, "Suction-Injection 
Effects on the Onset of Rayleigh-Bénard-Marangoni Convection in a Fluid with Suspended Particles," Acta Mechanica, Vol. 152, No. 1-4, 2001, pp. 241-252.

[27] P. G. Siddheshwar and S. Pranesh, "Magnetoconvection in Fluids with Suspended Particles under $1 \mathrm{~g}$ and $\mu \mathrm{G}$," International Journal of Aerospace Science and Technology, Vol. 6, No. 2, February 2001, pp. 105-114.

[28] P. G. Siddheshwar and S. Pranesh, "Effect of Tempera-
ture/Gravity Modulation on the Onset of Magneto Convection in Weak Electrically Conducting Fluids with Internal Angular Momentum," Journal of Magnetism and Magnetic Materials, Vol. 192, No. 1, February 1999, pp. 159-176.

[29] Lawrence C. Evans, "Partial Differential Equations (Schaum's Outline Series)," Tata McGraw Hill, India, 2010 . 